\title{
Application of Partial Differential Equations to Drum Head Vibration, Signal Transmission and Chemical Communication in Insects
}

\author{
Constance Atieno Ojwando \\ Department of Industrial and Engineering Mathematics, Technical University of Kenya, Nairobi, Kenya
}

\section{Email address:}

constatieno@yahoo.com

\author{
To cite this article: \\ Constance Atieno Ojwando. Application of Partial Differential Equations to Drum Head Vibration, Signal Transmission and Chemical \\ Communication in Insects. American Journal of Applied Mathematics. Vol. 4, No. 4, 2016, pp. 169-174. doi: 10.11648/j.ajam.20160404.11
}

Received: May 10, 2016; Accepted: May 28, 2016; Published: June 17, 2016

\begin{abstract}
The application of partial differential equations to drumhead vibration, signal transmission and chemical communication in insects has been investigated. This study looked at three cases in communication namely: a single drumbeat on an idealized circular drumhead being struck at the centre of the circular membrane; signal transmission in a Coaxial cable and finally concentration of pheromone in still air within a tunnel. The governing equation of Case I was solved using separation of variables method whereas Laplace transform was used to solve Cases II and III. MATLAB software was then used to obtain simulations on the solutions obtained from the three cases. It was concluded that vibrations of a circular drumhead as a result of a single drumbeat mainly concentrates around the centre of the membrane and tends to flatten out further away from the centre. Signal propagation decreases with increasing distance and so the shorter the cable the less signal strength is lost. Finally, concentration of pheromone can only last for a given time and distance.
\end{abstract}

Keywords: Cable, Vibration, Signal, Idealized Circular Drumhead, Pheromone Evaporation

\section{Introduction}

Humans gather most of our information in the outside world through sight and sound and relay the same information to the next party. In the process, we engage in communication. First, when we consider vibrations of a drumhead; it's worth noting that when the drum head is struck, the head vibrates in and out and physically displaces the air inside the drum. These vibrations create areas of high and low pressure and it's these changes in air pressure that creates the sound that we hear [1]. There exists infinitely many ways in which a drumhead can vibrate depending on the tension of the membrane, shape of the drum head, the number of times the drum is hit, the hitting point of the drum, the damping, the air pressure and the air volume inside the drum. [1] studied a Ghanaian drum; a drum that has strings holding the drumhead in place both from its upper and lower circles thus altering the tension on the drumhead. This deviates from the circular drum with constant tension on the drum head that possess both harmonic and rhythmic characteristics. [2] investigated the motion of a stretched elastic circular membrane which is subjected to a restorative force proportional to the velocity. [3] carried out a research on vibration of a Koch drum. He considered the vibrating membrane with fixed boundary and described the natural modes of vibrations by the eigen functions of the Laplacian on the region and then approximated the Koch drum with discrete grid of points. On the other hand [4] focused on the tone of a drum and observed that this tone is as a result of the oscillating vibrations of the drumhead attached to the drum shell. The oscillating drumhead creates vibrations within the drum which radiates outwards and is perceived as tone by our ears. Similarly [5] provided an algorithm of Elzaki projected differential transform method to solve the vibration equation of a very large membrane with given initial conditions.

Signal transmission takes place over a guided or unguided medium. The guided medium includes a coaxial cable, twisted pair cables and fiber-optic cable while the unguided medium is the wireless/free space (air, water, vacuum). This study focuses on a coaxial cable. According to [6], a coaxial cable is a transmission line consisting of two coaxial cylindrical conductors separated by a dielectric. In this paper 
we have referred to these coaxial cylindrical conductors as inner and outer conductors. [7] formulated a mathematical telegraph equation in terms of voltage and current for a section of transmission line. They solved the mathematical equation by reduced transform method under the assumption that the cable is imperfectly insulated so that there are both capacitance and current leakage to ground. In addition, [8] used finite difference approximations (second order difference method) in solving the passive cable equation by applying a current injection of $-0.01 \mu \cdot$ A with duration of 1 second to the cable at a single location and obtained the resulting voltage as a function of space and time.

Lastly, chemical signaling is the oldest and most widespread form of communication in animals. Some animals i.e. ants, honey-bee, and termites etc perceive nothing except a chemical world and all of their behavior is regulated by a relatively small number of substances (pheromone). In nature, chemical signals are used for intercellular and intra-cellular communication [9], whereas pheromones are used for long range communication between members of the same species such as social insects [10]. The chemical signals can therefore be used for communication at both microscopic and macroscopic scales. [11] carried out a research on mathematical modeling of ant pheromone: determination of optimum pheromone evaporation rate and simulation of pheromone tracking abilities. She investigated whether there is an optimum rate of pheromone evaporation when there is a certain probability of food randomly appearing in the foraging area. [12] worked on an individualbased model of ant-trail formation where the simulated ants followed a random walk process and modeled pheromone deposition and evaporation times by Poisson process. From these researches, it's evident that little investigations has been done on a single drumbeat on an idealized circular drumhead being struck at the centre of the circular membrane; signal transmission in a Coaxial cable and finally concentration of pheromone in still air within a tunnel. This study aimed at showing how diffusion and oscillatory effects plays an important role in communication. It finds its application in many real life situations related to communication in animals and human beings.

\section{Formulation of the Problem}

Case I: This case considers an idealized drum having a thin membrane stretched over a circular frame of unit radius. The vibrating membrane is radially symmetric and of constant tension. Its motion is governed by wave equation and so we will model a single drum beat using the $2 \mathrm{D}$ wave equation. Consider an open disk $\Omega$ of unit radius centered at the origin which represents the "still" drum head shape at any time t, the height of the drum head shape at a point $(\mathrm{x}, \mathrm{y})$ in $\Omega$ measured from the "still" drum head shape will be denoted by $\mathrm{u}(\mathrm{x}, \mathrm{y}, \mathrm{t})$, which can take both positive and negative values. Let $\partial \Omega$ denote the boundary of $\Omega$, i.e. circle of unit radius centered at the origin, which represents the rigid frame to which the drum head is attached. The mathematical equation that governs the vibration of the drum head is the $2 \mathrm{D}$ wave equation with zero boundary conditions.

$$
\frac{\partial^{2} \mathrm{u}}{\partial \mathrm{t}^{2}}=C^{2}\left(\frac{\partial^{2} \mathrm{u}}{\partial \mathrm{x}^{2}}+\frac{\partial^{2} \mathrm{u}}{\partial \mathrm{y}^{2}}\right) \text { for }(\mathrm{x}, \mathrm{y}) \epsilon \Omega ; \mathrm{u}=0 \text { on } \partial \Omega
$$

Due to the circular geometry of $\Omega$, we shall use the polar form of a $2 \mathrm{D}$ wave equation; where $\mathrm{x}=\mathrm{r} \cos \theta, \mathrm{y}=\mathrm{r} \sin \theta$ and $\theta=\tan ^{-1}\left(\frac{y}{x}\right)$. It is given by:

$$
\frac{\partial^{2} \mathrm{u}}{\partial \mathrm{t}^{2}}=C^{2}\left(\frac{\partial^{2} \mathrm{u}}{\partial \mathrm{r}}+\frac{1}{\mathrm{r}} \frac{\partial \mathrm{u}}{\partial \mathrm{r}}+\frac{1}{\mathrm{r}^{2}} \frac{\partial^{2} \mathrm{u}}{\partial \theta^{2}}\right)
$$

Since we are considering the vibration of a circular drumhead that is radlialy symmetric, the function $\mathrm{u}(\mathrm{r}, \mathrm{t})$ does not depend on the angle $\theta$. Therefore (2) reduces to

$$
\frac{\partial^{2} \mathrm{u}}{\partial \mathrm{t}^{2}}=C^{2}\left(\frac{\partial^{2} \mathrm{u}}{\partial \mathrm{r}}+\frac{1}{\mathrm{r}} \frac{\partial \mathrm{u}}{\partial \mathrm{r}}\right) \text { for } 0 \leq \mathrm{r} \leq 1
$$

$\mathrm{C}=\sqrt{ }\left(\frac{\mathrm{Nrr}}{\rho \mathrm{h}}\right)$ is a positive constant, which gives the speed at which transverse vibration waves propagate in the membrane.

Boundary Conditions: $u(r, t)=0$ on $\partial \Omega$ for all $t \geq 0 ; r=1$ since the drumhead is tautly held down at the boundary.

Initial conditions:

Initial displacement: $u(r, 0)=0$; its zero because the membrane is initially flat.

Initial velocity:

$$
\frac{\partial u}{\partial t}=\left\{\begin{array}{ll}
-\mathrm{V}_{0} & 0 \leq \mathrm{r} \leq \mathrm{b} \\
0 & \mathrm{~b} \leq \mathrm{r} \leq 1
\end{array} \text { at } \mathrm{t}=0\right\}
$$

Case II: Here, the researcher derived the equation for signal transmission in a cable using $\mathrm{x}$ to denote the longitudinal direction. The derivation was based on the assumptions that the potentials vary with distance along the line; that the transmitter is located at $\mathrm{x}=0$ and the receiver is at an arbitrary location $x$ up the line.

Consider some segment $(\mathrm{x}, \mathrm{x}+\mathrm{dx})$ along the inner and outer conductor of a cable. (see fig. 1).

Let $I_{0}(x), V_{0}(x), r_{0} I_{0}(x+d x)$ and $V_{0}(x+d x)$ be the current, voltage and resistance at some distances along the outer conductor and let $I_{i}(x), V_{i}(x), r_{i} I_{i}(x+d x) a n d V_{i}(x+$ $d x)$ be the current, voltage and resistance at some distances along the inner conductor. Let $C_{s} a n d r_{s}$ be the capacitance and resistance respectively. Ohms law for the segment ( $\mathrm{x}, \mathrm{x}$ $+\mathrm{dx}$ ) gives:

$$
\begin{aligned}
& V_{i}(\mathrm{x})-V_{i}(\mathrm{x}+\mathrm{dx})=I_{i}(\mathrm{x}) r_{i} \mathrm{dx} \\
& V_{0}(\mathrm{x})-V_{0}(\mathrm{x}+\mathrm{dx})=I_{0}(\mathrm{x}) r_{0} \mathrm{dx}
\end{aligned}
$$

Dividing through by $\mathrm{dx}$ in equations (4) and (5) and taking the limit as $d x \rightarrow 0$

$$
\begin{gathered}
\frac{\partial V_{i}}{\partial \mathrm{x}}=-r_{i} I_{i}(\mathrm{x}) \\
\frac{\partial V_{0}}{\partial \mathrm{x}}=-r_{0} I_{0}(\mathrm{x})
\end{gathered}
$$

Differentiating (6) and (7) w.r.t $\mathrm{x}$ 


$$
\begin{aligned}
& \frac{\partial^{2} \mathrm{Vi}}{\partial \mathrm{x}^{2}}=-\mathrm{r}_{\mathrm{i}} \frac{\partial \mathrm{Ii}}{\partial \mathrm{x}} \\
& \frac{\partial^{2} \mathrm{Vo}}{\partial \mathrm{x}^{2}}=-\mathrm{r}_{0} \frac{\partial \mathrm{Io}}{\partial \mathrm{x}}
\end{aligned}
$$

Subtracting (8) and (9)

$$
\frac{\partial^{2}(\mathrm{Vi}-\mathrm{Vo})}{\partial \mathrm{x}^{2}}=-\mathrm{ri} \frac{\partial \mathrm{Ii}}{\partial \mathrm{x}}+r_{0} \frac{\partial \mathrm{Io}}{\partial \mathrm{x}}
$$

Next we use the current conservation equation (Kirchoff's Law):

$$
\begin{aligned}
& I_{i}(\mathrm{x}+\mathrm{dx})=I_{i}(\mathrm{x})+I_{S}(\mathrm{x}) \mathrm{dx} \\
& I_{0}(\mathrm{x}+\mathrm{dx})=I_{0}(\mathrm{x})-I_{S}(\mathrm{x}) \mathrm{dx}
\end{aligned}
$$

Dividing through by $\mathrm{dx}$ in equations (11) and (12) and taking the limit as $\mathrm{dx} \rightarrow 0$, we obtain $\frac{\partial \mathrm{Ii}}{\partial \mathrm{x}}=I_{S}(\mathrm{x})$ and $\frac{\partial \mathrm{Io}}{\partial \mathrm{x}}=$ $-I_{S}(\mathrm{x})$ which implies that $I_{S}(\mathrm{x})=\frac{\partial \mathrm{Ii}}{\partial \mathrm{x}}=-\frac{\partial \mathrm{Io}}{\partial \mathrm{x}}$. Inserting this in (10) and redefining the transinsulator potential as $\mathrm{V}=V_{i}-V_{0}$, (10) now becomes

$$
\frac{\partial^{2} \mathrm{~V}}{\partial \mathrm{x}^{2}}=-\left(\mathrm{r}_{\mathrm{i}}+\mathrm{r}_{0}\right) \operatorname{Is}(\mathrm{x})
$$

Or

$$
\frac{\frac{\partial^{2} \mathrm{v}}{\partial \mathrm{x}^{2}}}{\left(\mathrm{r}_{\mathrm{i}}+\mathrm{r}_{0}\right)}=-I_{S}(\mathrm{x})
$$

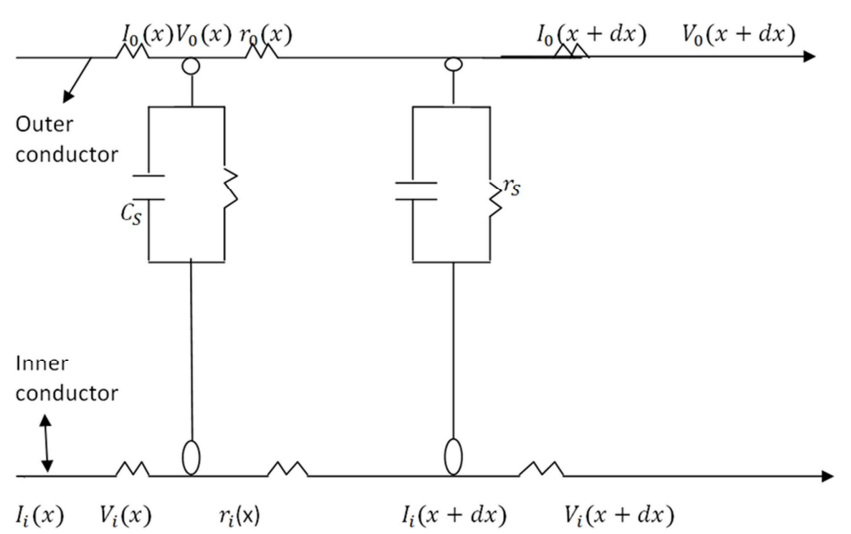

Figure 1. Longitudinal cross-section of a Coaxial Cable.

If we define the current $I_{S}(\mathrm{x})$ as the sum of the contribution made by the resistor $r_{s}:\left(\frac{-1}{r_{s}}\right) \mathrm{V}$ and the current through a capacitor $\left(-\operatorname{Cs} \frac{\partial \mathrm{V}}{\partial \mathrm{t}}\right)$. (13) then becomes $\frac{\frac{\partial^{2} \mathrm{~V}}{\partial \mathrm{x}^{2}}}{\left(\mathrm{r}_{\mathrm{i}}+\mathrm{r}_{0}\right)}=$ $\frac{\mathrm{v}}{\mathrm{r}_{\mathrm{S}}}+\mathrm{C}_{\mathrm{S}} \frac{\partial \mathrm{v}}{\partial \mathrm{t}}$ Multiply through by $\mathrm{r}_{\mathrm{S}}$ and divide through by $\mathrm{r}_{\mathrm{S}} \mathrm{C}_{\mathrm{S}}$,

$$
\frac{1}{\operatorname{Cs}\left(\mathrm{r}_{\mathrm{i}}+\mathrm{r}_{0}\right)} \frac{\partial^{2} \mathrm{~V}}{\partial \mathrm{x}^{2}}=\frac{\mathrm{V}}{\mathrm{r}_{\mathrm{S}} \mathrm{Cs}}+\frac{\partial \mathrm{V}}{\partial \mathrm{t}}
$$

This becomes $\mathrm{D} \frac{\partial^{2} \mathrm{~V}}{\partial \mathrm{x}^{2}}=\beta \mathrm{V}+\frac{\partial \mathrm{V}}{\partial \mathrm{t}}$ where $\mathrm{D}=\frac{1}{\operatorname{Cs}\left(\mathrm{r}_{\mathrm{i}}+\mathrm{r}_{0}\right)}$ and $\beta=\frac{1}{r_{S} C s}$ where $\beta$ is the loss of energy between the inner and the outer conductor); which gives us the governing equation for $0<x<\infty$ and $-\infty<t<\infty$

Boundary and Initial conditions:

- $\mathrm{V}(0, \mathrm{t})=\mathrm{A} \cos \omega t$ to account for the fact that we need an oscillatory data when solving this signal transmission equation at $\mathrm{x}=0$

- $\mathrm{V}(\mathrm{x}, \mathrm{t}) \rightarrow 0$ as $\mathrm{x} \rightarrow \infty$ since the transinsulator potential diminishes with increase in distance from the transmitter.

- $\mathrm{V}(\mathrm{x}, 0)=0$ since the transinsulator potential is initially zero.

Case III: Since the transmission of chemical signals (pheromones) takes place by the process of diffusion in which the random movement of molecules transports the chemical away from its source, we solved the 1D diffusion equation under the assumptions that diffusion is in the direction of the ants locomotion (x-axis), the release of pheromones lasts for a sufficiently long time and that the effects of wind and turbulence are ignored.

Consider an ant emitting an alarm chemical into the still air of a tunnel. Let $C(x, t)$ denote the concentration of the chemical $\mathrm{xcm}$ from the source at time $\mathrm{t}$, then the governing equation is

$\frac{\partial \mathrm{C}}{\partial \mathrm{t}}=\mathrm{D} \frac{\partial^{2} \mathrm{C}}{\partial x^{2}}, \mathrm{x}>0, \mathrm{t}>0$; where $\mathrm{D}$ is the diffusion coefficient. This equation models the flow of chemicals on the ground at a time $t$ on emission by an ant.

Boundary conditions:

- $\frac{\partial \mathrm{C}}{\partial \mathrm{t}}=-\mathrm{A} \delta \mathrm{t}$ at $\mathrm{x}=0, \mathrm{~A}>0$, where $\delta \mathrm{t}$ is the Dirac delta function. This negative is imposed because pheromone concentration falls in still air. A $\delta \mathrm{t}$ is a constant because in this study we consider release of pheromone as a discrete pulse.

- $C(x, 0)=0, x>0$ since initially there is no release of pheromone in the tunnel.

- $\lim _{x \rightarrow \infty} C(x, t)=0, \mathrm{t}>0$ since the concentration of pheromone in still air diminishes with increasing distance from the source and the signal no longer elicits a response at an infinitely longer distance.

\section{Analytical Solutions}

The set of differential equations in the three cases were solved analytically subject to the various boundary and initial conditions stated for each case. Case I was solved using separation of variables method while Case IIand III were solved using Laplace transform method.

Case I:

Consider the equation

$$
\frac{\partial^{2} \mathrm{u}}{\partial \mathrm{t}^{2}}=C^{2}\left(\frac{\partial^{2} \mathrm{u}}{\partial \mathrm{r}^{2}}+\frac{1}{\mathrm{r}} \frac{\partial \mathrm{u}}{\partial \mathrm{r}}\right) \text { for } 0 \leq r \leq 1
$$

and let

$$
\mathrm{U}(\mathrm{r}, \mathrm{t})=\mathrm{R}(\mathrm{r}) \mathrm{T}(\mathrm{t})
$$

Differentiating (16) w.r.t $\mathrm{r}$ and $\mathrm{t}$ and inserting in (3)

$$
\mathrm{RT}^{\prime \prime}=C^{2}\left(\mathrm{R}^{\prime \prime} \mathrm{T}+\frac{1}{\mathrm{r}} \mathrm{R}^{\prime} \mathrm{T}\right) \text { or }
$$




$$
\frac{1}{C^{2}} \frac{\mathrm{T}^{\prime \prime}}{\mathrm{T}}=\frac{\mathrm{R}^{\prime \prime}+\frac{\mathrm{R} \prime}{\mathrm{r}}}{\mathrm{R}}
$$

Since the LHS of (17) does not depend on r, and RHS does not depend on $t$, both sides must be equal to some constant say $-\mu$ (separation constant).

Then

$$
\mathrm{T}^{\prime \prime}+\mu C^{2} \mathrm{~T}=0
$$

(simple harmonic oscillator equation).

Next we solve (18); we shall consider three cases of $\mu$

i) $\mu=0$

$\mathrm{T}^{\prime \prime}=0 ; \mathrm{T}(\mathrm{t})=\mathrm{At}+\mathrm{B}$

This is a linear case that does not depict our oscillatory physical situation, so we reject it.

ii) $\mu<0$; $=-\alpha^{2}$

$\mathrm{T}^{\prime \prime}-\alpha^{2} c^{2} \mathrm{~T}=0 ; m^{2}=\alpha^{2} c^{2}, \mathrm{~m}= \pm \alpha \mathrm{c}$

$\mathrm{T}(\mathrm{t})=\mathrm{C} e^{\alpha C t}+\mathrm{D} e^{-\alpha C t}$. This solution show exponential growth or decay that does not depict our physical situation, we reject it too.

iii) $\mu>0 ;=\alpha^{2}$

$\mathrm{T}^{\prime \prime}+\alpha^{2} c^{2} \mathrm{~T}=0 ; m^{2}+\alpha^{2} c=0 ; \mathrm{m}= \pm \mathrm{i} \alpha \mathrm{c}$

$T(\mathrm{t})=\mathrm{ECos}(\alpha \mathrm{Ct})+\mathrm{FS} i n(\alpha \mathrm{Ct})$. Applying the B.C: $\mathrm{U}(\mathrm{r}, 0)=0$ implying that $\mathrm{T}(0)=0$ but $\mathrm{E}=0$ therefore

\section{$\mathrm{T}(\mathrm{t})=\mathrm{F} \sin \alpha C t$}

This gives us the required solution.

Rewriting the RHS of (17) using $-\mu$, we obtain: $r^{2} \mathrm{R}^{\prime \prime}+$ $\mathrm{rR}^{\prime}+\mu r^{2} \mathrm{R}=0$ or

$$
r^{2} \mathrm{R}^{\prime \prime}+\mathrm{rR}^{\prime}+\alpha^{2} r^{2} \mathrm{R}=0
$$

Which is an example of Bessel's equation. We use Bessel's equation in this situation because according to [13] they are useful when dealing with circular geometry and can be expanded as an infinite series. The solution to (20) is $\mathrm{R}(\mathrm{r})=\mathrm{G} J_{0}(\alpha \mathrm{r})+Y_{0}(\alpha \mathrm{r})$. But for our case, the $Y_{0}$ functions are not allowed as $Y_{0} \rightarrow \infty$ asr $\rightarrow 0$. Only $J_{0}$ functions are allowed since they are finite at $r=0$. We therefore have:

$$
\mathrm{R}(\mathrm{r})=J_{0}(\alpha \mathrm{r})
$$

Combining (19) and (21) and applying the Superposition Principle, we obtain the solution

$$
\begin{gathered}
\mathrm{U}(\mathrm{r}, \mathrm{t})=\sum_{n=1}^{\infty}\left(H_{n} \sin \beta_{n} \mathrm{Ct}\right) J_{0}\left(\beta_{n} \mathrm{r}\right) \\
\text { Or } \mathrm{U}(\mathrm{r}, \mathrm{t})=\sum_{n=1}^{\infty}\left(H_{n} \sin \beta_{n} \mathrm{Ct}\right) J_{0}\left(\beta_{n} \mathrm{r}\right)
\end{gathered}
$$

Applying the initial condition:

$$
\frac{\partial u}{\partial t}=g(r)=\left\{\begin{array}{ll}
-\mathrm{V}_{0} & 0 \leq \mathrm{r} \leq \mathrm{b} \\
0 & \mathrm{~b} \leq \mathrm{r} \leq 1
\end{array} \text { at } \mathrm{t}=0\right\}
$$

Differentiating (22) w.r.t $\mathrm{t}$ and inserting $\mathrm{t}=0$

$\mathrm{g}(\mathrm{r})=\sum_{n=1}^{\infty}\left(\mathrm{H}_{\mathrm{n}} \beta_{\mathrm{n}} \mathrm{C}\right) \mathrm{J}_{0} \beta_{\mathrm{n}} \mathrm{r}$. Next we apply Fourier Bessel Series and use Bessel's recurrence relation to obtain the value of $H_{n}$, the amplitude of displacement of the drumhead.

Rewriting (22) we get the solution as:

$U(r, t)=\frac{-2 v 0 b}{C} \sum_{n=1}^{\infty} \frac{J 1\left(\beta_{n} b\right)}{\left.\beta_{n}{ }^{2} J_{1}{ }^{2} \beta_{n}\right)} \sin \beta_{n} C t J_{0}\left(\beta_{n} r\right) \quad$. The values of $J_{0}\left(\beta_{\mathrm{n}}\right)$ for $\mathrm{n}=1,2,3,4$ were used in MATLAB to obtain various simulations of circular drumhead vibration for different values of $t$.

Case II:

Consider the passive cable equation

$$
\mathrm{D} \frac{\partial^{2} \mathrm{~V}}{\partial \mathrm{x}^{2}}=\beta \mathrm{V}+\frac{\partial \mathrm{V}}{\partial \mathrm{t}}
$$

Applying Laplace transform to both sides We obtain $\mathrm{s} \bar{V}(\mathrm{x}, \mathrm{s})-\mathrm{V}(\mathrm{x}, 0)=\mathrm{D} \frac{\partial^{2} \overline{\mathrm{V}}}{\partial \mathrm{x}^{2}}-\beta \bar{V}(\mathrm{x}, \mathrm{s}) \quad$ but $\quad \mathrm{V}(\mathrm{x}, 0)=$ 0 , so $\mathrm{D} \frac{\partial^{2} \overline{\mathrm{v}}}{\partial \mathrm{x}^{2}}-\left(\frac{\beta+\mathrm{s}}{\mathrm{D}}\right) \bar{V}=0$.

Upon solving we get

$$
\begin{aligned}
& \bar{V}(\mathrm{x}, \mathrm{s})=C_{1} e^{\sqrt{\left(\frac{\beta+s}{D}\right) x}}+C_{2} e^{-\sqrt{ }\left(\frac{\beta+s}{D}\right) x} \\
& \mathrm{~V}(\mathrm{x}, \mathrm{t}) \rightarrow 0 \text { as } x \rightarrow \infty ; \text { so } 0=C_{1} e^{\infty}+C_{2} e^{-\infty}
\end{aligned}
$$

We fix $C_{1}=0$ to avoid the exponential from growing infinitely. So (23) now reduces to

$$
\bar{V}(\mathrm{x}, \mathrm{s})=C_{2} e^{-\sqrt{ }\left(\frac{\beta+s}{D}\right) x}
$$

Next we find the Laplace transform of the first boundary condition.

$$
\mathrm{L}[\mathrm{V}(0, \mathrm{t})]=\mathrm{AL}(\cos \omega \mathrm{t})=\mathrm{A} \frac{\mathrm{s}}{\omega^{2}+\mathrm{s}^{2}}
$$

Hence (24) reduces to

$$
\bar{V}(\mathrm{x}, \mathrm{s})=e^{-\sqrt{ }\left(\frac{\beta}{D}\right) x} \frac{\mathrm{s}}{\omega^{2}+\mathrm{s}^{2}} e^{-\sqrt{ }\left(\frac{s}{D}\right) x}
$$

Lastly we get the Laplace inverse of (25)

$$
\begin{array}{r}
\mathrm{V}(\mathrm{x}, \mathrm{t})=\mathrm{A} e^{-\sqrt{ }\left(\frac{\beta}{D}\right) x} L^{-1}\left\{\frac{\mathrm{s}}{\omega^{2}+\mathrm{s}^{2}} e^{-\sqrt{ }\left(\frac{s}{D}\right) x}\right\} \\
=A e^{-\sqrt{ }\left(\frac{\beta}{D}\right) x} \cos \left(\omega \mathrm{t}-x^{2} \mathrm{D}\right) .
\end{array}
$$

The solution to this problem is therefore $\mathrm{V}(\mathrm{x}, \mathrm{t})=$ $\mathrm{A} e^{-\sqrt{ }\left(\frac{\beta}{D}\right) x} \cos \left(\omega \mathrm{t}-x^{2} \mathrm{D}\right)$.

where $\sqrt{ }\left(\frac{\beta}{D}\right)$ shows how exponential amplitude decay as the signal travels down the line.

Case III: In this case, the researcher uses Laplace transform to solve

$$
\frac{\partial \mathrm{C}}{\partial \mathrm{t}}=\mathrm{D} \frac{\partial^{2} \mathrm{C}}{\partial x^{2}}, \mathrm{x}>0, \mathrm{t}>0
$$

Applying Laplace on both sides of (26), we obtain

$$
\mathrm{s} \bar{C}(\mathrm{x}, \mathrm{s})-\mathrm{C}(\mathrm{x}, 0)=\mathrm{K} \frac{\partial^{2} \bar{C}}{\partial x^{2}}
$$

But $\mathrm{C}(\mathrm{x}, 0)=0$ from the third B.C as stated in Case III - 
problem formulation above.

$$
\mathrm{K} \frac{\partial^{2} \bar{C}}{\partial x^{2}}-\mathrm{s} \bar{C}(\mathrm{x}, \mathrm{s})=0
$$

It's solution is $\bar{C}(\mathrm{x}, \mathrm{s})=C_{1} e^{\sqrt{ }\left(\frac{S}{K}\right) x}+C_{2} e^{-\sqrt{ }\left(\frac{S}{K}\right) x}$

Using the next B.C: $\lim _{x \rightarrow \infty} \mathrm{C}(\mathrm{x}, \mathrm{t})=0$, we obtain $0=C_{1} e^{\infty}+C_{2} e^{-\infty}$. But since the exponential must not be allowed to grow indefinitely, we make $C_{1}=0$ so we now have

$$
\bar{C}(\mathrm{x}, \mathrm{s})=C_{2} e^{-\sqrt{ }\left(\frac{S}{K}\right) x}
$$

Differentiating (27) w.r.t x;

$$
\frac{\mathrm{d} \bar{C}}{\mathrm{dx}}=-C_{2} \sqrt{ }\left(\frac{S}{K}\right) e^{-\sqrt{ }\left(\frac{S}{K}\right) x}
$$

Next we find the Laplace transform of the initial condition as stated in Case III under problem formulation above

$$
\begin{gathered}
\mathrm{L}\left\{\frac{\partial \mathrm{C}}{\partial \mathrm{x}}\right\}=-\mathrm{AL}[\delta \mathrm{t}] \text { but } \mathrm{L}[\delta \mathrm{t}]=1 \\
\frac{\mathrm{d} \bar{C}}{\mathrm{dx}}=-\mathrm{A} \text { at } \mathrm{x}=0
\end{gathered}
$$

(27) now becomes $\bar{C}(\mathrm{x}, \mathrm{s})=\mathrm{A} \sqrt{ }\left(\frac{S}{K}\right) e^{-\sqrt{ }\left(\frac{K}{S}\right) x}$

On applying Laplace inverse the final solution becomes

$$
\mathrm{C}(\mathrm{x}, \mathrm{s})=\sqrt{ }\left(\frac{K}{\pi t}\right) e^{\sqrt{\left(\frac{-x^{2}}{4 k t}\right)}}
$$

\section{Discussion of Results}
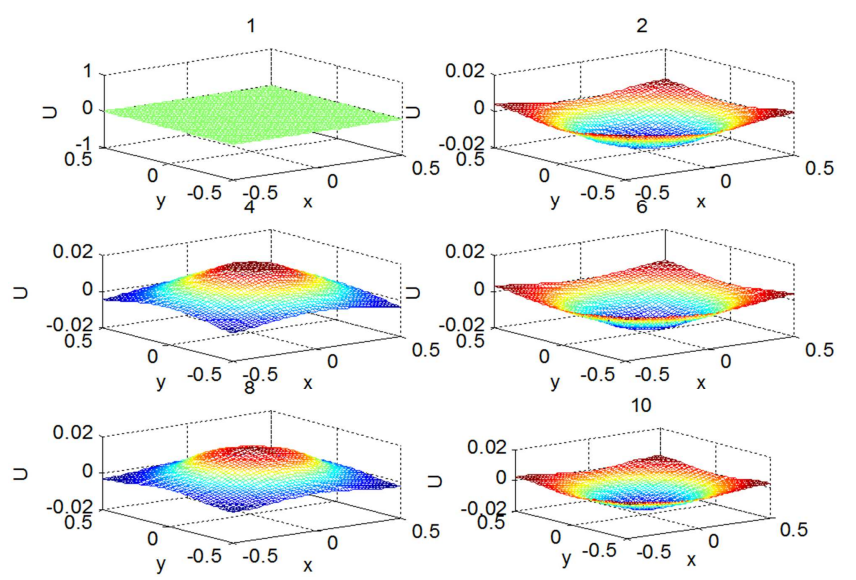

Figure 2. Simulations on drumhead vibration at different times.

Key: $\mathrm{t}=1$ stands for the time before the membrane is hit, $\mathrm{t}=2,4,6,8$ and 10 shows the membrane's vibration after $2 \mathrm{~s}, 4 \mathrm{~s}, 6 \mathrm{~s}, 8 \mathrm{~s}$ and $10 \mathrm{~s}$ respectively.

CASE I, from the simulations in figure 2 above, the researcher concluded that there is no vibration of the membrane initially since the membrane is initially flat. When a slight force is applied (single drumbeat) at the centre of this circular drumhead, there is a slight deflection of the membrane due to the pressure exerted on the membrane; a single drumbeat causes vibrations to be localized around the centre of the drum (hitting point) and the membrane gradually flattens at a distance further away from the centre. This was observed for all times $\mathrm{t}=2 \mathrm{~s}, 4 \mathrm{~s}$, $6 \mathrm{~s}, 8 \mathrm{~s}, 10 \mathrm{~s}$.

\section{CASE II}
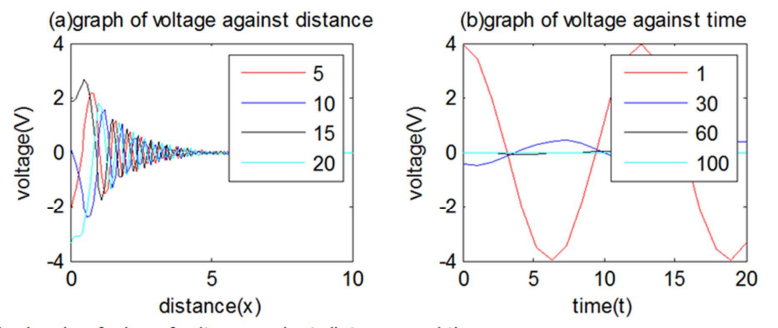

(c)graph showing fusion of voltage against distance and time

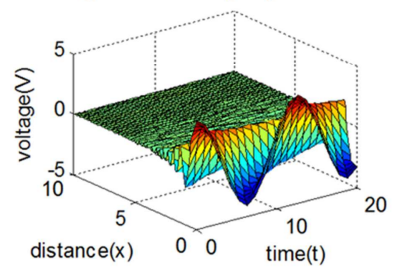

Figure 3. Signal propagation with changing distance and time.

Figure 3, (a) shows that for all time t, the signal strength decreases with increasing distance. The longer the distance the signal travels, the more it gets attenuated and finally after propagating through a long distance, the signal gets vanished completely, (b) depicts the fact that there is distortion as signal is being transmitted for different distance travelled for all $t$, (c) gives how signal transmission varies with both distance and time. It was also noted that the closer the distance is to the transmitter $(\mathrm{x}=0)$, the faster the signal propagation. This explains the reason why normally receivers are put close to the transmitters.

CASE III:

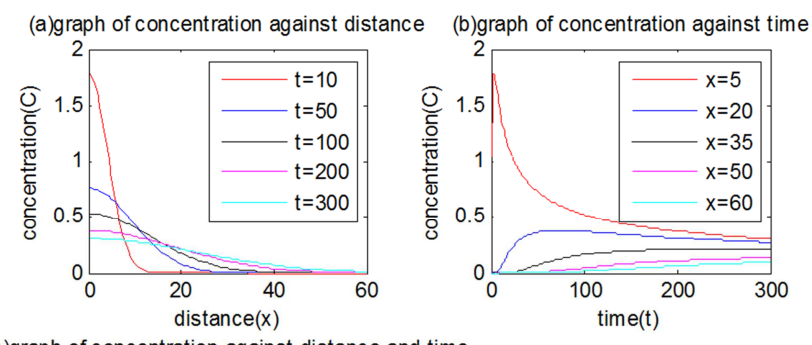

(c)graph of concentration against distance and time

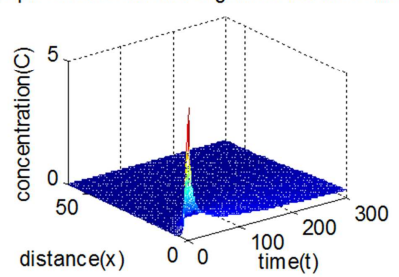

Figure 4. Showing pheromone travelling at different times and distance.

Finally, for Case III it was noted that the shorter the time the pheromone travels along the tunnel the higher the concentration of pheromone within the channel. Figure 4(a) 
shows that at all times, pheromone concentration decreases gradually with increasing distance and later stabilizes at some further distance from the source. This implies that ants no more receive the alarm message at a given distance from the source; (a) implies that the concentration of pheromone is generally greater at a distance closer to the source of the pheromone itself; (b) shows that pheromone concentration within the tunnel is higher at shorter times and decreases as time increases. (c) depicts the fact that concentration increases and reaches its maximum at some given distance and time; then it decreases gradually as a result of evaporation and diffusion of the pheromone along the tunnel as both distance and time increases. After some times, the concentration falls below the threshold value throughout the tunnel and the signal no longer elicits a response.

\section{Conclusion}

It was concluded that vibrations of a circular drumhead as a result of a single drumbeat mainly concentrates around the centre of the membrane and tends to flatten out further away from the centre. For the case of signal propagation, we concluded that signal propagation decreases with increasing distance and so the shorter the cable the less signal strength is lost. Finally, concentration of pheromone can only last for a given time and distance. Once the concentration reaches some particular time and distance, the ants can no longer receive any signal since the pheromonal path will have disappeared completely.

\section{References}

[1] G. O. Lartey \& A. Adu. Sackey, The mathematics of Ghanaian Circular Musical Drumheads: varying tension versus constant tension. Journal of Science and Technology, Vol. 33, No 2(2013) pp 81-87.

[2] Doma S. B, Sirafy I. H, Borai M. M and El-Sharif A. H
Pertubation treatment for the vibrations of a circular membrane subject to a restorative force, Alexandria Journal of Mathematics, Vol. 1, No 1, June 2010.

[3] Mark C, Vibration of the Koch drums. Mathematica in Education and Research, 12(2), 2007.

[4] Marshall P, Drumkit / Drumsetmechanics and construction, Drum Dojo. http://www.drumdojo.con/kit/equipment.htm (2000).

[5] Muhammad Suleiman, Qingbiao Wu, T. M. Elzaki, Explicit Analytic Solution of Vibration Equation for large domain by mean of the Elzaki projected differential transform method. Journal of Advances in Mathematics, Vol. 11, No 5, October $13,2015$.

[6] Renarto Orta, Lecture notes on transmission line theory. November 2012.

[7] Vineet K. Srivasta, Mukesh K. Awasthi, R. K Chaurasia \& M. Tamsir, The Telegraph Equation and its Solution by Reduced Differential Transform Method, Hindawi Publishing Corporation, Modelling \& Simulation in engineering. Vol. 2013, Article ID 746351.

[8] Distasio M (2010), Numerical solutions to the passive Cable equation: using finite difference approximations to partial derivatives. Published Thesis.

[9] B. Alberts, A. Johnson, J. Lewis, M. Raff, K. Roberts \& P. Walter. Molecular Biology of the Cell. $5^{\text {th }}$ edition, Garland Sc. November 2007.

[10] W. C. Agosta, Chemical Communication: The Language of Pheromones, 1st edition. W, H. Freeman \& Co. August 1992.

[11] Katie K, Mathematical modeling of Ant pheromone: Determination of optimum pheromone evaporation rate and simulation of pheromone tracking abilities. (2008) Unpublished Thesis.

[12] Bollsard E, Degond P and Motsh S, Trail formation based on directed pheromone deposition-September 15,2011; Thesis.

[13] B. Neta, Partial Differential Equations, MA 3132 Lecture Notes, Naval Postgraduate School, Monterey California 2012. 